\title{
Charge Decay Measurements on a Polymeric Insulation Material under Controlled Humidity Conditions
}

\author{
vonne p ck arath umara tanislaw M. ubanski \\ High oltage ngineering Chalmers niversity of Technology othenburg weden \\ Comesponding uthor spaeck hst.tu-darmstadt.de
}

\begin{abstract}
bstract-
In this work a systematic study on the influence of relative humidity on the charge decay of high-temperature-vulcanized flat silicone rubber samples is carried out by means of contactless measurements of the surface potential. Both virgin and aged (by a long-term corona treatment) samples were used for the experiments. Temporal and spatial variations of the potential were monitored during the charge decay process. The analysis of the results indicated that the charge decay becomes faster due to aging and/or presence of humidity in the surrounding air.
\end{abstract}

\section{I T D CTI}

Il over the world more and more sustainable energy resources are utilized due to an increasing environmental consciousness but also due to growing energy consumption. s some of those sources are located far away from load centers long-distance electric power transmission operating at ultra high voltage ( $\mathrm{H}$ ) levels needs to be further developed. or such $H$ applications use of polymeric based insulation instead of traditional glass and porcelain insulators provides both technical and economical benefits. However, published research results on this field provide evidence that charge carriers deposited on the surface of polymeric insulation may influence the flash-over performance [1], [2], especially under direct current (DC) conditions. Thus studying the surface charge decay on polymeric surfaces under various atmospheric conditions is essential to overcome this obstacle.

everal studies reported faster charge decay due to higher moisture content in surrounding gaseous media if polymeric material has a significant surface conduction [3], [4], [5]. Moreover in humid atmospheres Crisci et al. considered surface conduction among the leading decay mechanisms. utz et al. state that the relative humidity $(\mathbf{H})$ in the gaseous environment seems to strongly influence the charge decay characteristics. However, influence of moisture content on charge decay on materials having dominant bulk conductivity compared to surface conduction has not been addressed.

The present work systematically studies the influence of moisture content on the charge decay of virgin and aged silicone rubber material sample surfaces. The measured surface potential decay and distributions were utilized to analyze the influence of moisture content on charge decay due to surface and/or bulk conduction.

\section{II. $\mathrm{CH}$ \\ D $\mathrm{C} \quad \mathrm{M}$ CH I $\mathrm{M}$}

Previous studies related to charge decay on thick insulation material samples proposed three main mechanisms governing the decay process namely surface conduction as well as gas and bulk neutralization

- urface charges deposited on a flat insulation sample placed on a grounded plane can decay due to various bulk related processes such as intrinsic conduction of the bulk material charge in ection at gas-solid interface and polarization processes . If charge decay is solely governed by these processes resulting potential decay characteristics can be used to derive bulk conductivity which is an apparent conductivity representing all bulk related processes [6], [8]. Firstly, the field-dependent bulk conductivity $\sigma(\quad)$ can be expressed as

$$
\sigma(\quad)-\frac{\epsilon}{(\mathbf{t})} \frac{\mathbf{d}(\mathbf{t})}{\mathbf{d t}}
$$

where $\epsilon$ denotes the permittivity and the potential. The calculated bulk conductivity can further be represented according to the Poole-Frenkel model which is well accepted for fielddependent bulk conductivities in polymeric materials

$$
\sigma(\quad) \quad \sigma \exp \left(\beta^{\mathbf{p}}-\right)
$$

In this model $\beta$ represents the Poole-Frenkel coefficient, which is dependent on temperature permittivity and thickness of the insulation sample .

In presence of surface conduction deposited charges are transported along the surface i.e. gas-solid interface of the insulation material and results in surface charge decay at least in some regions on the surface. This neutralization mode strongly depends on the conditions of the surrounding gaseous environment, such as pollution or $\mathrm{RH}$ and is significantly increased for aged material [3], [6], [9]. Influence of surface conduction can be seen in both spatial and temporal surface charge distributions.

Charge carriers present in a surrounding gaseous environment may be attracted to the surface due to the field created by the surface charge. pon reaching the surface these charge camiers neutralize the surface charges. This mode of charge decay is specified as gas neutralization [10]. In this work, the charge decay due to gas neutralization is suppressed due to the chosen measurement approach cf. .

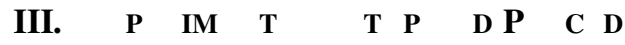

igure shows the experimental setup used to deposit charges on the sample surfaces and to measure the resulting surface potentials. The material sample is kept under open circuit configuration, i.e. one surface on a grounded copper plate while the opposite surface is open to the surrounding gaseous environment. 


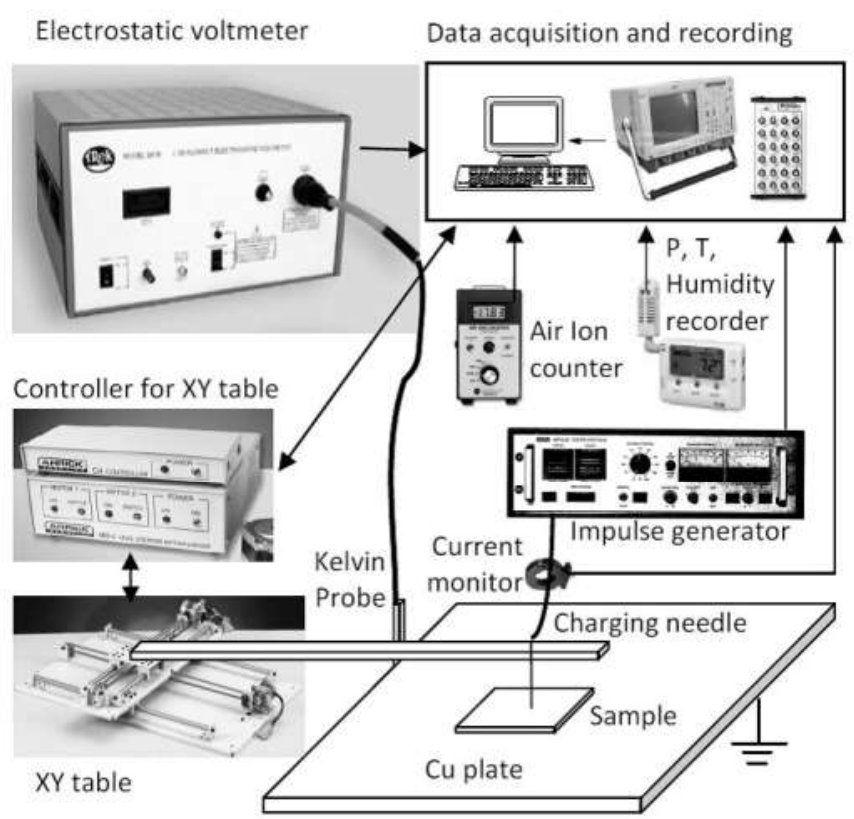

igure - chematic diagram of the experimental setup

Before starting the measurement procedure the material samples were cleaned to remove residual charges. fter waiting a certain time for drying an initial surface potential scan was conducted to prove a proper neutralization of the sample. fter that the sample was charged with a needle ( . mm in diameter) positioned in the middle of the sample mounted mm above its surface. The Haefely $P$ impulse generator was used to provide standard lightning impulse voltage ( . I $\mu \mathrm{s}$ ) with the peak value of $\mathbf{k}$. During charging both the applied voltage and charging current were recorded. fter the charging a linear positioning system moved the elvin type probe (Trek ) which was connected to a electrostatic voltmeter (Trek b) to the middle of the sample. sing a data ac uisition system the measured surface potential was recorded at a sample rate of $\mathrm{Hz}$ in order to achieve information on the time-dependency of the charge decay. To monitor the spatial distribution of the charges on the sample surface a surface scanning along a diametrical line on the surface was undertaken. This scan was repeated several times in order to monitor the decay process over time. Preliminary measurements showed a rotational symmetrical charging spot thus allowing to perform the surface scan only along a diametrical line. In addition to surface potential the ambient conditions such as relative humidity temperature and pressure were recorded by a measuring system ( $\mathrm{T}$ - $)$ installed close to the samples.

The investigated samples ( $\left.\begin{array}{cccc}\mathbf{m m} \mathbf{x} & \mathbf{m m} \mathbf{x} & \mathbf{m m}\end{array}\right)$ are high-temperature-vulcanized flat silicone rubber materials with relative permittivity of . at Hz. Two virgin and two aged samples were tested. ging was achieved by $h$ long conona treatment as described in . The experiments were performed in a humidity chamber made of acrylic glass that covers the whole experimental setup with the dimensions of approx. $\quad \mathbf{m m} \mathbf{x} \quad \mathbf{m m} \mathbf{x} \quad \mathbf{m m}$. The humidity control was established using different saturated salt dilutions according to. Three levels of $\mathbf{H}$ were studied (i)

$\mathbf{H}$ (without salt dilutions i.e. ambient condition) (ii) $\quad \mathbf{H}$ i.e. dry condition and (iii) $\quad H$ i.e. wet condition. fter establishing the salt dilution and reaching stable conditions the surface charging and potential measurement procedure was started. or these materials acclimatization time was in the range of - $h$. Due to the large volume of the humidity chamber once stabilized at a certain value comesponding to type of the salt the $H$ was very stable and robust against disturbances such as short term opening for sample exchange. During these experiments ambient temperature remained fairly constant in the range of $-{ }^{\circ} \mathrm{C}$.

$$
\text { I . T D DI C I }
$$

- Comparison of untreated material in different humidity conditions

irstly we analyze the charge decay characteristics on the untreated samples (i.e. virgin) under three humidity levels. This step of the work is motivated by examining the $\mathbf{H}$ influence on the untreated material bulk conductivity $\sigma$.

igure a shows a spatial potential distribution along a diametrical line on the surface. The distribution displays saddleshape characteristic. ccording to this appearance can be attributed to back discharges between the charged surface and the needle electrode. evertheless the distribution shows a sufficient radial symmetry. Moreover, analyzing the spatial distribution in igure a yields the following results

- charged region is spread up to about . - cm

- not having a lateral expansion of the charged region during the decay indicates that the decay process is mainly governed by bulk neutralization without significant contribution from surface conduction cf.

- comparatively faster charge decay in regions of high potential, e.g. sample's center, confirms existence of fielddependent decay-process in the material bulk cf.

In conclusion no surface conduction was detected in am-

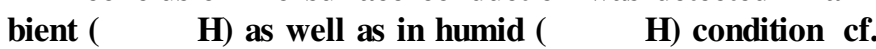
igure c. Hence no surface conduction is expected for the dry condition of $\quad H$. This assumption of based on Crisci et al. and utz et al. who detect an increasing surface conduction in more humid conditions. Hence no scans have been performed at $\mathrm{H}$.

fter the exclusion of surface conduction it is valid to calculate the bulk conductivity of the material based on the presented data using uation ( ). igure depicts the variation of the calculated conductivity versus the $s$ uare root of the surface potential in for each $H$ level.

e observe a linear decline of the graphs from high surface

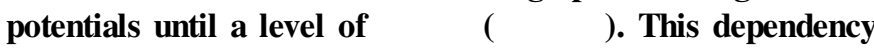
is referred to as Poole- renkel type behavior as introduced in uation ( ). The second part of the curve shows a rapid reduction of $\sigma$ it seems likely that the designated model does not hold for describing this part of the decay probably due to the low electric field strength. 


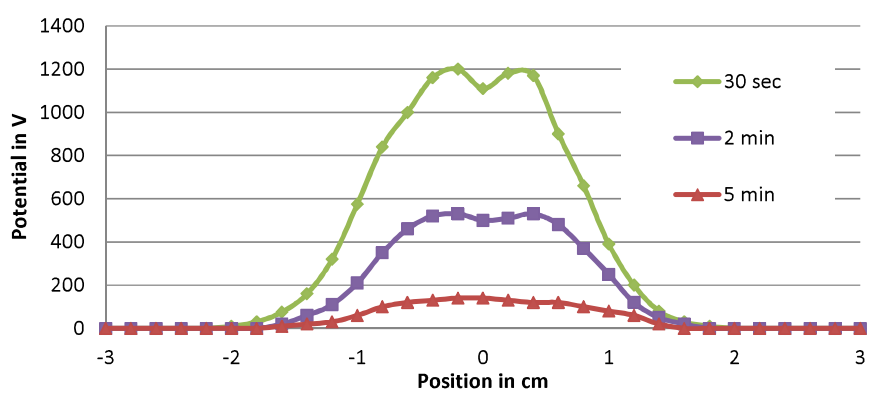

(a) ntreated material at $\mathrm{H}$

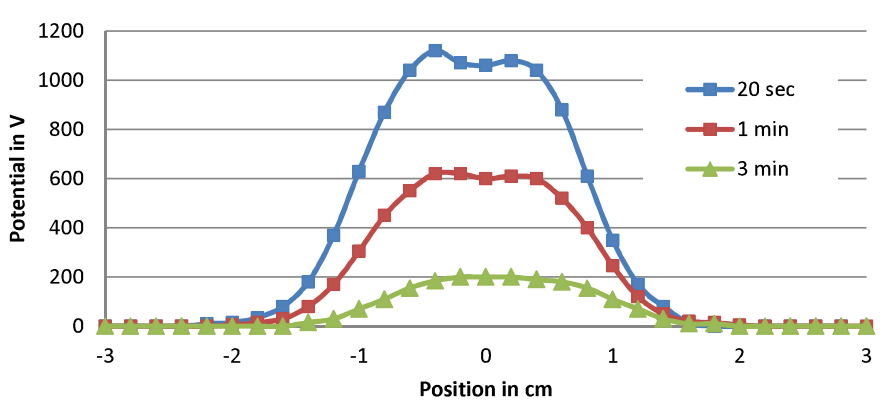

(c) ntreated material at

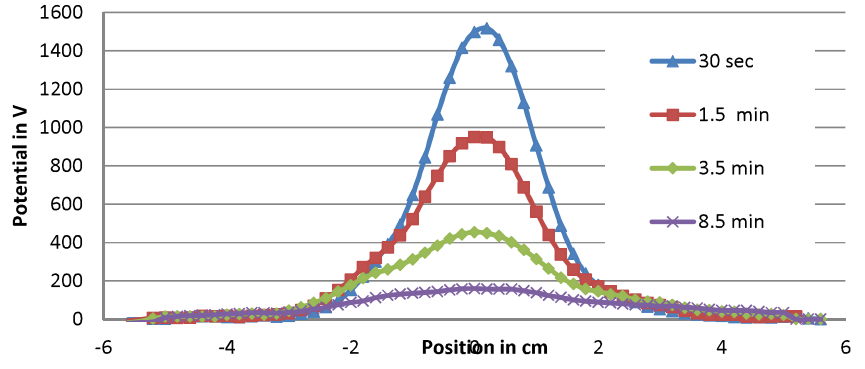

(b) Treated material at $\mathrm{H}$

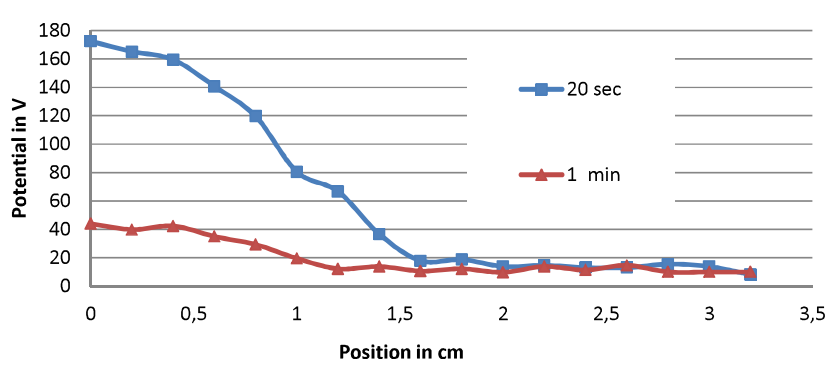

(d) Treated material at $\quad \mathrm{H}$

igure - patial distribution of surface potential at different $\mathbf{H}$ conditions

or each $H$ condition the shape of the graphs shows great similarity. uniform scaling of the conductivity over all surface potentials can be observed. ne can conclude that a change of the humidity conditions yields a increase of the conductivity levels. Besides, a significantly higher conductivity for higher $H$ can be observed (for ) at $\mathbf{H} \quad{ }^{-} \mathbf{m}^{-}$compared to $\cdot{ }^{-} \mathbf{m}^{-}$ at H. imilar characteristics can be observed even at moderately lower potential values eg for at $\mathbf{H}$ $\mathbf{m}^{-}$compared to ${ }^{-} \mathbf{m}^{-}$at $\mathbf{H}$ can be detected which again differ in one order of magnitude. However increasing $\mathrm{H}$ from to $\mathrm{H}$ does not significantly affect the conductivity levels.

In conclusion, the influence of the RH level on the untreated material where bulk conduction is the dominant charge decay mechanism is evident.

B. Comparison of treated material under different humidity conditions

This section summarizes and contrasts the charge decay characteristics for treated and untreated material under different humidity conditions.

irstly an analysis of the spatial charge distribution is carried out. igures $b$ and $d$ present the measurement at and $\quad H$ respectively. Due to fast charge decay dynamic at $\quad H$ the surface potential scan was limited to a radial line starting from the sample center. $t$ both $H$ levels the charge distribution leads to a bell-shaped potential distribution. The initially symmetric charging spot with a large diameter (already approx. cm after $s$ ) in igure $b$ at $\quad H$ is

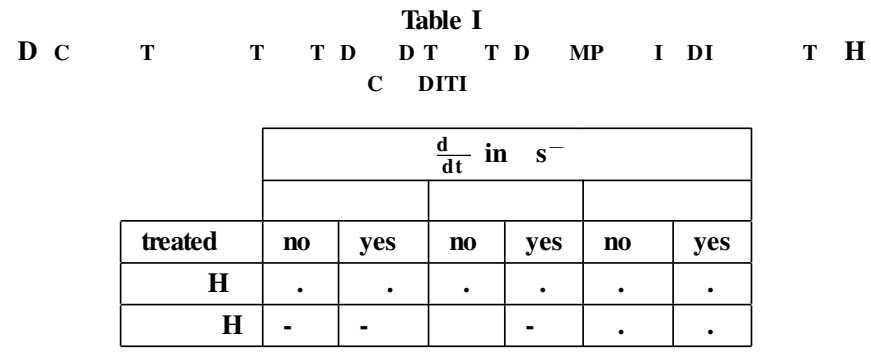

dispersed over the whole surface during the measurement at - min . min and . min. This observed charge transport along the sample surface indicates contribution of surface conduction to the charge decay. ote that in the case of

$H$ it was not possible to charge the samples to higher potentials as for other cases. Due to this lower initial ( $\mathbf{s}$ after charging) potentials disturbances from the measuring system clutter the radial scan in igure d. evertheless one can detect an initial bell-like potential distribution that decays rapidly. Again, the influence of surface conduction seems to cause a spread of charges over the whole recorded range of cm.

Due to the appearance of surface conduction it is not valid to calculate the bulk conductivity based on the measurement data. Hence the decay rate is selected as comparison parameter since it is a measure of the dynamic in the decay process according to uation ( ).

igure presents the calculated decay rates for untreated and treated material at different humidity conditions. ddition- 


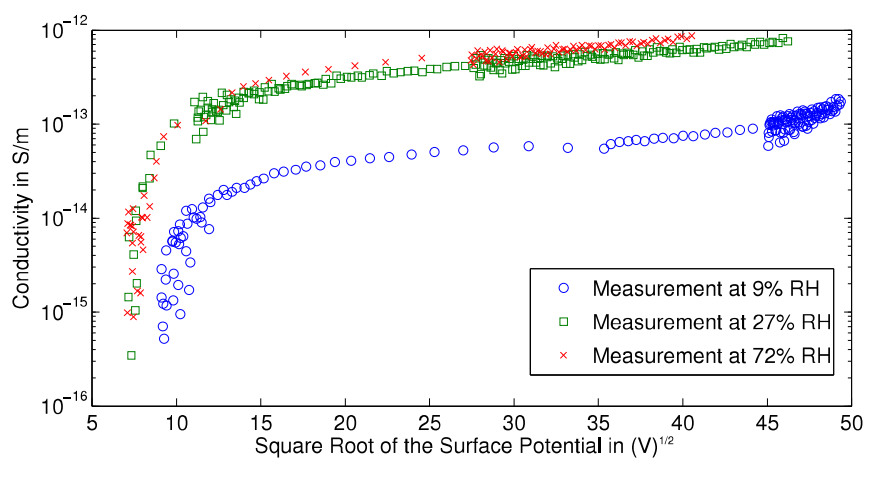

igure . Calculated conductivities at different $\mathrm{H}$ levels

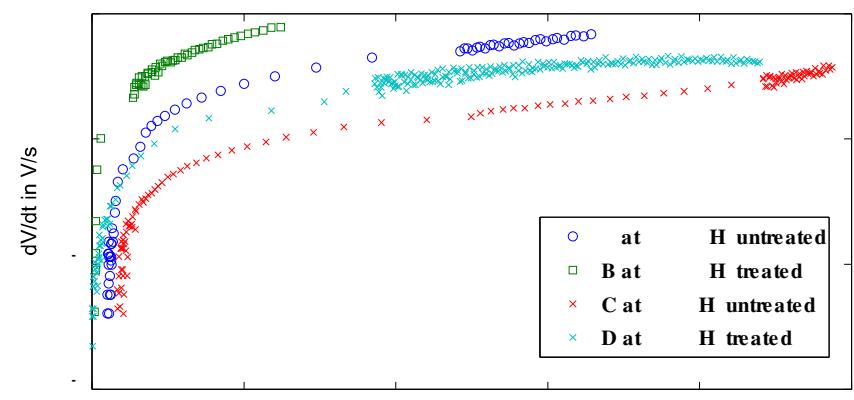

urface Potential in

igure - Comparison of potential decay rate for untreated and treated material under different humidity conditions

ally Table I lists the decay rate at selected surface potential levels. irstly one can observe that throughout the decay process its rate seems to be persistent see ig. - rapid reduction of the decay rate occurs at about . Thus we conclude that the fundamental coherence of decay rate is not influenced by humidity and/or aging.

t higher potential levels ( ) the magnitude of the decay rate depends on the test conditions i.e on aging and the humidity content. or example in case of untreated materials increase of $\mathrm{H}$ from to results times higher decay rate. The discrepancy of the decay rate due to humidity is even more pronounced for the treated material. $t$ a level of

$H$ results in a decay rate of . $s^{-}$in contrast to - $\mathbf{s}^{-}$at which is an increment by a factor of .

Moreover the treatment results in fast decay dynamics. nder dry conditions the increment e uals approx. - times e.g. . $s^{-}$for untreated compared to.$\quad s^{-}$at a level of . This is primarily due to increased surface conduction in the case of treated samples. nder humid conditions this difference is more pronounced for the treatment increased the decay rate from . $\mathbf{s}^{-}$to. $\mathbf{s}^{-}$.

In summary, both of the modified conditions, $\mathrm{RH}$ and treatment, influence the charge decay dynamics significantly.

lthough the fundamental voltage dependency of the decay rate is persistent a pronounced increase in the decay rate can be observed if the $H$ level is increased or the material has been sub ected to aging treatment. Moreover the presence of surface conduction for the treated material contributes to enhanced decay dynamics.

$$
\text { - C C I }
$$

In this work the charge decay on polymeric insulation material under controlled humidity conditions was experimentally investigated. nalysis of the surface potential decay on virgin and aged samples at various moisture contents revealed that the charge decay dynamics for the silicone rubber samples are strongly influenced by moisture content and aging. A Significant rise of the decay rate on aged sample can be attributed to an increased surface conduction. aster charge decay at higher relative humidity level is caused by higher volume and surface conduction for the untreated and treated samples respectively. The difference in the decay rate of untreated vs. treated material is more pronounced in a humid ( $\mathrm{H})$ environment. The presence of surface conduction for the treated material may partly account for the enhanced decay dynamics.

- umara - endyuk and - ubanski imulation of surface charge effect on impulse flashover characteristics of outdoor polymeric insulators I Transactions on Dielectrics and lectrical Insulation vol. no. pp. dec.

- umara - lam I. Ho ue - endyuk and - ubanski DC flashover characteristics of a polymeric insulator in presence of surface charges I Transactions on Dielectrics and lectrical Insulation vol. no. pp. une .

- Crisci B. osse .-P. osse and . llier-Dur ault urfacepotential decay due to surface conduction The uropean Physical ournal pplied Physics vol. pp.

- umara lectrical charges on polymeric insulator surfaces and their impact on withstand performance. Chalmers niversity of Technology

[5] B. Lutz and J. Kindersberger, "Influence of relative humidity on surface charge decay on epoxy resin insulators I th International Conference on the Properties and pplications of Dielectric Materials vol. pp.

- umara . . endyuk and . M. ubanski urface charge decay on polymeric materials under different neutralization modes in air I Transactions on Dielectrics and lectrical Insulation vol. no. pp.

- umara - erdyuk and . M. ubanski urface charge decay of corona charged htv silicone nubber samples due to bulk conduction in nd ordic Insulation ymposium ( DI ) Tampere inland une - pp.

H. stedt . . endyuk and . M. ubanski Charging characteristics of PDM and silicone rubbers deduced from surface potential measurements I Transactions on Dielectrics and lectrical Insulation vol. no. pp.

C. ederte and . indersberger urface charge decay on insulators in air and sulfurhexafluorid - part i: Simulation," I Transactions on Dielectrics and lectrical Insulation vol. no. pp.

H. Stedt Electric charges on insulator surfaces and their influence on insulator performance ser. Technical report (Department of Materials and Manufacturing Technology Chalmers niversity of Technology). Chalmers niversity of Technology .

B. Ma valuating resistance to corona and ozone in polymeric materials for high voltage outdoor applications. Chalmers niversity of Technology

[12] L. Greenspan, "Humidity fixed points of binary saturated aqueous solutions Physics vol. no. pp. 